\title{
MARTINGALE CONVERGENCE THEOREMS FOR SEQUENCES OF STONE ALGEBRAS
}

\author{
by J. D. MAITLAND WRIGHT
}

(Received 12 January, 1968)

1. Introduction. A vector lattice $W$ is boundedly complete when each subset $\left\{a_{j}: j \in J\right\}$ of $W$ which is bounded above by an element of $W$ has a least upper bound in $W$. The least upper bound of $\left\{a_{j}: j \in J\right\}$ is denoted by $\bigvee_{j \in J} a_{j}$ and the greatest lower bound by $\bigwedge_{j \in J} a_{j}$ whenever these exist.

Let $C(S)$ be the algebra of real valued continuous functions on a compact Hausdorff space $S$. Stone [4] shows that the vector lattice $C(S)$ is boundedly complete if and only if the closure of each open subset of $S$ is open; in this event we call $C(S)$ a Stone algebra. For example, if $(X, \mathscr{B}, \mu)$ is a probability space, then $L^{\infty}(X, \mathscr{B}, \mu)$ is a Stone algebra satisfying the countable chain condition.

Let $\left\{a_{n}\right\}(n=1,2, \ldots)$ be a bounded sequence in a Stone algebra $\mathscr{S}$; then

$$
\bigvee_{n=1}^{\infty} \bigwedge_{r=n}^{\infty} a_{r} \leqq \bigwedge_{n=1}^{\infty} \bigvee_{r=n}^{\infty} a_{r}
$$

When these two terms are equal we define $\operatorname{LIM} a_{n}$ to be their common value and say the sequence is order convergent with order limit $\operatorname{LIM} a_{n}$. In the special case where $\mathscr{S}$ is of the form $L^{\infty}(X, \mathscr{B}, \mu)$ and $\mu$ is a probability measure, if a sequence $\left\{b_{n}\right\}(n=1,2, \ldots)$ has order limit $b$, then the sequence $\left\{b_{n}\right\}(n=1,2, \ldots)$ converges to $b$ in the $L^{1}$-topology ( $L^{\infty}$ is the dual of $L^{1}$ ). But Floyd [3] gives an example of a Stone algebra $\mathscr{S}$ such that there is no Hausdorff vector topology for $\mathscr{S}$ in which each bounded monotone increasing sequence converges to its least upper bound.

We shall postpone all further definitions till §2. In [7] we investigated Moy averaging operators on Stone algebras satisfying the countable chain condition. In this paper we consider a monotone increasing sequence $\left\{\mathscr{A}_{n}\right\}(n=1,2, \ldots)$ of Stone subalgebras of a Stone algebra $\mathscr{A}_{\infty}$ such that the smallest Stone subalgebra containing $\bigcup_{n=1}^{\infty} \mathscr{A}_{n}$ is $\mathscr{A}_{\infty}$. Let $\mathscr{A}_{\infty}$ satisfy the countable chain condition and let $T_{0}: \mathscr{A}_{\infty} \rightarrow \mathscr{A}_{1}$ be a Moy operator satisfying certain conditions. Then we show that there exists a sequence $\left\{T_{n}\right\}(n=1,2, \ldots)$ of Moy operators on $\mathscr{A}_{\infty}$ such that:

(i) $T_{n}$ is a projection of $\mathscr{A}_{\infty}$ onto $\mathscr{A}_{n}$ for $n \geqq 1$.

(ii) $T_{r} T_{n}=T_{r}$ for $0 \leqq r<n$.

(iii) If $b$ is a positive element of $\mathscr{A}_{\infty}$ and $T_{n} b=0$ then $b=0$.

(iv) For each $z \in \mathscr{A}_{\infty}$ the order limit $\operatorname{LIM} T_{n} z$ exists and $\operatorname{LIM} T_{n} z=z$.

This result is a Corollary of Theorem 2.

Theorem 1 is a convergence theorem for a sequence of generalized conditional expectations with respect to a modular Stone algebra valued measure. For conditional expectations with respect to real valued measures such results are known in probability theory as martingale 
theorems; see Doob [2]. Theorem 1 was suggested by the classical work of Sparre Andersen and Jessen in [1]. The key step in generalizing their result to Stone algebra valued measures is Lemma 1.

In a later publication I intend to discuss applications of the results of this paper to Boolean algebras.

The work of this paper depends essentially on that of [6]. This is because in [7] we used the results of [6] to establish the existence, under certain conditions, of generalized conditional expectations.

2. Convergence theorems. Throughout this paper $(X, \mathscr{B})$ is a measurable space and $C(S)$ is a Stone algebra. Stone algebra valued measures were defined in [5]. We require $\rho$ to be a finite $C(S)$-valued measure on $(X, \mathscr{B})$; that is, $\rho$ is to be a map of $\mathscr{B}$ into $C(S)$ such that

(i) $\rho E \geqq 0$ for each $E \in \mathscr{B}$;

(ii) if $\left\{E_{j}\right\}(j=1,2, \ldots)$ is a pairwise disjoint family of sets in $\mathscr{B}$ then

$$
\rho \bigcup_{j=1}^{\infty} E_{j}=\bigvee_{n=1}^{\infty} \sum_{j=1}^{n} \rho E_{j} .
$$

A Stone algebra $\mathscr{S}$ satisfies the countable chain condition when each bounded subset of $\mathscr{S}$ contains a countable subset such that the two sets have the same least upper bound. This condition on $\mathscr{S}$ is equivalent (see Proposition 3.2 of [6]) to the Boolean algebra of idempotent elements of $\mathscr{S}$ satisfying the countable chain condition. From now onward we require $C(S)$ to satisfy the countable chain condition.

We defined $L^{p}$-spaces with respect to Stone algebras in [6], and it follows from Proposition 3.3 that $L^{\infty}(X, \mathscr{B}, \rho)$ is a Stone algebra satisfying the countable chain condition because $C(S)$ satisfies this condition.

We require the existence of an algebra homomorphism $\pi: C(S) \rightarrow L^{\infty}(X, \mathscr{B}, \rho)$ such that

$$
\int_{X} \pi(a) f d \rho=a \int_{X} f d \rho \text { for each } f \in \mathscr{L}^{1}(X, \mathscr{B}, \rho) .
$$

Then $\rho$ is a modular measure with respect to $\pi$, as defined in [6]. Close connections between modular measures and averaging operators were exhibited in [7].

Let $\mathscr{S}$ be a Stone algebra and $\mathscr{U}$ a subalgebra. $\mathscr{U}$ is a Stone subalgebra of $\mathscr{S}$, if the least upper bound, in $\mathscr{S}$, of each upper bounded subset of $\mathscr{U}$ is in $\mathscr{U}$; i.e. $\mathscr{U}$ is a Stone algebra and a bounded subset of $\mathscr{U}$ has the same least upper bound in $\mathscr{S}$ and $\mathscr{U}$.

Let $T$ be a linear operator on a Stone algebra $\mathscr{S} . \quad T$ is an averaging operator if $T(f T g)=(T f)(T g)$ for each $f$ and $g$ in $\mathscr{S}$. T is a Moy averaging operator when $T$ is a positive averaging operator and, if $\left\{f_{n}\right\}(n=1,2, \ldots)$ is a monotone increasing sequence in $\mathscr{S}$ which is bounded above, then $T \bigvee_{n=1}^{\infty} f_{n}=\bigvee_{n=1}^{\infty} T f_{n}$. For any operator $T$ on $\mathscr{S}$ let

$$
\mathscr{E}(T)=\{a \in \mathscr{S}: a T b=T a b \text { for all } b \in \mathscr{S}\} \text {. }
$$

When $T$ is an averaging operator the range of $T$ is a subset of $\mathscr{E}(T)$. It is shown in [7] that when $T$ is a Moy operator and $\mathscr{S}$ satisfies the countable chain condition then $\mathscr{E}(T)$ is a Stone subalgebra of $\mathscr{S}$. 
When $\mathscr{B}_{1}$ is a Boolean $\sigma$-subalgebra of $\mathscr{B}$ and $\rho_{1}$ is the restriction of $\rho$ to $\mathscr{B}_{1}$ then $L^{\infty}\left(X, \mathscr{B}_{1}, \rho_{1}\right)$ can be identified with a Stone subalgebra of $L^{\infty}(X, \mathscr{B}, \rho)$. If $\pi[C(S)]$ is a subalgebra of $L^{\infty}\left(X, \mathscr{B}_{1}, \rho_{1}\right)$, then for each $f \in \mathscr{L}^{1}(X, \mathscr{B}, \rho)$ we can find a $\mathscr{B}_{1}$-measureable function $f_{1} \in \mathscr{L}^{1}\left(X, \mathscr{B}_{1}, \rho_{1}\right)$ such that

$$
\int_{E} f d \rho=\int_{E} f_{1} d \rho_{1} \quad \text { for each } E \in \mathscr{B}_{1} .
$$

This is Lemma 2.1 of [7].

DefintTION 1. Let $\mathscr{B}_{1}$ be a $\sigma$-subalgebra of $\mathscr{B}$ such that $\pi[C(S)] \subset L^{\infty}\left(X, \mathscr{B}_{1}, \rho_{1}\right)$. The conditional expectation of $\mathscr{B}_{1}$ with respect to $\rho$ is the map $C: L^{1}(X, \mathscr{B}, \rho) \rightarrow L^{1}\left(X, \mathscr{B}_{1}, \rho_{1}\right)$ such that for each $[f]_{\rho} \in L^{1}(X, \mathscr{B}, \rho)$ we have $C[f]_{\rho}=\left[f_{1}\right]_{\rho}$, where

$$
\int_{E} f d \rho=\int_{E} f_{1} d \rho \text { for each } E \in \mathscr{B}_{1} .
$$

The generalized conditional expectation operator $C$, defined above, is a positive linear map of $L^{1}(X, \mathscr{B}, \rho)$ onto $L^{1}\left(X, \mathscr{B}_{1}, \rho_{1}\right)$ such that $C^{2}=C$. The restriction of $C$ to $L^{\infty}(X, \mathscr{B}, \rho)$ is a Moy averaging operator whose range is $L^{\infty}\left(X, \mathscr{B}_{1}, \rho_{1}\right)$.

LEMMA 1. Let $\mathscr{W}$ be a Boolean subalgebra of $\mathscr{B}$ such that $\mathscr{B}$ is the smallest $\sigma$-algebra of subsets of $X$ containing $\mathscr{V}$. Let $f \in \mathscr{L}^{1}(X, \mathscr{B}, \rho)$ be such that $\int_{E} f d \rho \geqq 0$ for each $E \in \mathscr{W}$. Then $\int_{E} f d \rho \geqq 0$ for each $E \in \mathscr{B}$.

Proof. Let $\mathscr{U}=\left\{E \in \mathscr{B}: \int_{E} f d \rho \geqq 0\right\}$; then by hypothesis $\mathscr{W} \subset \mathscr{U}$. An argument using Zorn's lemma shows that there is a maximal Boolean algebra $\mathscr{M}$ such that $\mathscr{W} \subset \mathscr{M} \subset \mathscr{U}$.

Let $\mathscr{M}^{*}=\left\{E \subset X: \chi_{E}=\lim \chi_{E_{n}}\right.$, where each $\left.E_{n} \in \mathscr{M}\right\}$, so that $\mathscr{M} \subset \mathscr{M}^{*}$. If $A \in \mathscr{M}^{*}$ and $B \in \mathscr{M}^{*}$ then $A \cap B$ and $X-A$ are in $\mathscr{M}^{*}$. Hence $\mathscr{M}^{*}$ is a Boolean algebra containing $\mathscr{M}$.

Let $E \in \mathscr{M}^{*} ;$ then $\chi_{E}=\lim \chi_{E_{n}}$, where $E_{n} \in \mathscr{M}$ for each $n$. Then, by the analogue for Stone algebra valued measures of the Dominated Convergence Theorem established in [5], we have

$$
\int_{E} f d \rho=\int_{X} f \chi_{E} d \rho=\operatorname{LIM} \int_{X} f \chi_{E_{n}} d \rho=\operatorname{LIM} \int_{E_{n}} f d \rho
$$

Thus $\int_{E} f d \rho \geqq 0$ and so $\mathscr{M}^{*} \subset \mathscr{U}$. It now follows from the maximality of $\mathscr{M}$ that $\mathscr{M}=\mathscr{M}^{*}$. Thus $\mathscr{M}$ is a Boolean $\sigma$-algebra containing $\mathscr{W}$ and thus $\mathscr{M}=\mathscr{U}=\mathscr{B}$.

THEOREM 1. Suppose that $\rho$ is a finite $C(S)$-valued measure on the measurable space $(X, \mathscr{B})$ and suppose that $\rho$ is modular with respect to $\pi$. Let $\left\{\mathscr{B}_{n}\right\}(n=1,2, \ldots)$ be a monotone increasing sequence of $\sigma$-subalgebras of $\mathscr{B}$ such that $\mathscr{B}$ is the smallest $\sigma$-subalgebra of $\mathscr{B}$ containing $\bigcup_{n=1}^{\infty} \mathscr{B}_{n}$. Further, let $\pi[C(S)]$ be a subalgebra of $L^{\infty}\left(X, \mathscr{B}_{1}, \rho\right)$. For each $n$ let $T_{n}$ be the 
generalized conditional expectation of $\mathscr{B}_{n}$ with respect to $\rho$. Let $f \in \mathscr{L}^{1}(X, \mathscr{B}, \rho)$ and let $f_{n} \in L^{1}\left(X, \mathscr{B}_{n}, \rho\right)$ be such that $\left[f_{n}\right]_{\rho}=T_{n}[f]_{\rho}$ for each $n$. Then $\lim f_{n}(x)=f(x)$ almost everywhere with respect to $\rho$.

Proof. The set $F=\left\{x \in X: \underline{\lim } f_{n}(x)<f(x)\right\}$ is the countable union of all sets of the form

$$
F_{\alpha, \beta}=\left\{x \in X: \underline{\lim } f_{n}(x) \leqq \alpha<\beta \leqq f(x)\right\},
$$

where $\alpha$ and $\beta$ are rational and $\alpha<\beta$. Assume that $\rho F \neq 0$; then $\rho F_{\lambda, \mu} \neq 0$ for some rational numbers $\lambda$ and $\mu, \lambda<\mu$.

Let $L_{\lambda}=\left\{x \in X: \underline{\lim } f_{n}(x) \leqq \lambda\right\}$ and, for each natural number $n$, let

$$
H_{n}=\left\{x \in X: \inf _{r>n} f_{r}(x)<\lambda+\frac{1}{n}\right\}
$$

Let

and, for $q \geqq 2$,

$$
H_{n, 1}=\left\{x \in X: f_{n+1}(x)<\lambda+\frac{1}{n}\right\}
$$

$$
H_{n, q}=\left\{x \in X: \min \left\{f_{r}(x): n<r<n+q\right\} \geqq \lambda+\frac{1}{n} \text { and } f_{n+q}(x)<\lambda+\frac{1}{n}\right\} \text {. }
$$

Since $f_{n+q}$ is $\mathscr{B}_{n+q}$-measurable, $H_{n, q} \in \mathscr{B}_{n+q}$. Also $\left\{H_{n, q}\right\} \quad(q=1,2, \ldots)$ is a pairwise disjoint family such that $H_{n}=\bigcup_{q=1}^{\infty} H_{n, q}$. We also have $L_{\lambda}=\bigcap_{n=1}^{\infty} H_{n}$.

Choose $A \in \bigcup_{1}^{\infty} \mathscr{B}_{n}$, so that $A \in \mathscr{B}_{N}$ for some $N$. Then $H_{n, q} \cap A \in \mathscr{B}_{n+q}$ for $n \geqq N$ and $q \geqq 1$. By Proposition 3.3 of [6]

$$
\int_{A} f \chi_{H_{n}} d \rho=\operatorname{LIM} \int_{A} \sum_{q=1}^{r} f \chi_{H_{n, q}} d \rho .
$$

From the definition of $T_{n+q}$ and $f_{n+q}$ we have, for $n \geqq N$,

So

$$
\begin{aligned}
\int_{A} f \chi_{H_{n, q}} d \rho & =\int_{A \cap B_{n, q}} f_{n+q} d \rho \\
& \leqq\left(\lambda+\frac{1}{n}\right) \int_{A} \chi_{H_{n, q}} d \rho .
\end{aligned}
$$

Thus

$$
\int_{A} f \chi_{H_{n}} d \rho \leqq\left(\lambda+\frac{1}{n}\right) \int_{A} \chi_{H_{n}} d \rho \text { for } n \geqq N \text {. }
$$

$$
\int_{A}\left(\lambda+\frac{1}{n}\right) \chi_{H_{n}}-f \chi_{H_{n}} d \rho \geqq 0 \text { for } n \geqq N .
$$

But $\lim _{n} \chi_{H_{n}}=\chi_{L_{\lambda}}$ and so 


$$
\lim _{n}\left(\lambda+\frac{1}{n}\right) \chi_{H_{n}}-f \chi_{H_{n}}=(\lambda-f) \chi_{L_{\lambda}} .
$$

So, by Proposition 3.5 of [6],

$$
\int_{A}(\lambda-f) \chi_{L_{\lambda}} d \rho \geqq 0 \text { for each } A \in \bigcup_{n=1}^{\infty} \mathscr{B}_{n} \text {. }
$$

We observe that $\bigcup_{n=1}^{\infty} \mathscr{B}_{n}$ is a Boolean subalgebra of $\mathscr{B}$ and, by hypothesis, $\mathscr{B}$ is the $\sigma$ algebra generated by $\bigcup_{n=1}^{\infty} \mathscr{B}_{n}$. It now follows from Lemma 1 that

$$
\int_{A}(\lambda-f) \chi_{L_{\lambda}} d \rho \geqq 0 \text { for each } A \in \mathscr{B} .
$$

We replace $A$ by $F_{\lambda, \mu}$ in the above inequality and since $F_{\lambda, \mu} \subset L_{\lambda}$, obtain

$$
\lambda \rho F_{\lambda, \mu} \geqq \int_{F_{\lambda, \mu}} f d \rho \geqq \mu \rho F_{\lambda, \mu} .
$$

Since $\mu>\lambda$ this implies that $F_{\lambda, \mu}=0$. This is a contradiction; so the assumption $\rho F \neq 0$ must be false. Thus $f(x) \leqq \lim f_{n}(x)$ for almost all $x$.

Applying this result to $-f$ we obtain $f(x) \geqq \overline{\lim } f_{n}(x)$ for almost all $x$.

So $\lim f_{n}$ exists and equals $f$ almost everywhere with respect to $\rho$.

We now strip away the measure theory of Theorem 1 and obtain the following abstract martingale theorem.

THEOREM 2. Let $\left\{\mathscr{A}_{n}\right\}(n=1,2, \ldots)$ be an increasing sequence of Stone subalgebras of a Stone algebra $\mathscr{A}_{\infty}$ such that the smallest Stone subalgebra containing $\bigcup_{n=1}^{\infty} \mathscr{A}_{n}$ is the whole of $\mathscr{A}_{\infty}$. Let $\mathscr{A}_{0}$ be a Stone algebra satisfying the countable chain condition and $\pi: \mathscr{A}_{0} \rightarrow \mathscr{A}_{1}$ an algebra homomorphism. Let $T_{0}: \mathscr{A}_{\infty} \rightarrow \mathscr{A}_{0}$ be a positive linear map such that:

(i) If $b \geqq 0$ and $T_{0} b=0$ then $b=0$.

(ii) $T_{0}(\pi(a) z)=a T_{0} z$ for each $z \in \mathscr{A}_{\infty}$ and each $a \in \mathscr{A}_{0}$.

(iii) If $\left\{z_{n}\right\}(n=1,2, \ldots)$ is a bounded monotone increasing sequence of positive elements of $\mathscr{A}_{\infty}$ then

$$
T_{0}\left(\bigvee_{n=1}^{\infty} z_{n}\right)=\bigvee_{n=1}^{\infty} T_{0} z_{n}
$$

Then there exists a sequence of Moy operators $\left\{T_{n}\right\}(n=1,2, \ldots)$ such that:

(i) $T_{n}$ is a projection of $\mathscr{A}_{\infty}$ onto $\mathscr{A}_{n}$ for each $n \geqq 1$.

(ii) If $b \geqq 0$ and $T_{n} b=0$ then $b=0$.

(iii) $T_{r} T_{n}=T_{r}$ for $0 \leqq r<n$.

(iv) For each $z \in \mathscr{A}_{\infty}$ the order limit $\operatorname{LIM} T_{n} z$ exists and $\operatorname{LIM} T_{n} z=z$.

Proof. Let $\mathscr{A}_{\infty} \cong C(E)$, the ring of continuous functions on an extremally disconnected compact Hausdorff space $E$. For each Borel set $A$ in $E$ there is a unique idempotent $k(A)$ 
in $C(E)$ which differs from $\chi_{A}$ only on a meagre Borel set. We recall from [5] that $k$ is a $C(E)$-valued measure, the map $f \rightarrow \int_{E} f d k$ is an algebra homomorphism of $B^{\infty}(E)$ (the bounded Borel functions on $E$ ) onto $C(E)$ and the kernel of this homomorphism is the set of Borel functions vanishing outside a meagre Borel set.

Let $m$ be defined on the Borel sets of $E$ by $m B=T_{0}(k B)$. Then $m$ is a (finite) $\mathscr{A}_{0}$-valued measure on the Borel sets of $E$ and for each $f \in B^{\infty}(E)$ we have

$$
\int_{E} f d m=T_{0}\left(\int_{E} f d k\right)
$$

Let $B$ be any Borel set of $E$; then $m B=0$ if and only if $k B=0$, that is, if and only if $B$ is meagre. Thus

$$
L^{\infty}(E, m) \cong C(E) \cong \mathscr{A}_{\infty} .
$$

For each $a \in \mathscr{A}_{0}$ and $f \in B^{\infty}(E)$ we have

But, by hypothesis,

$$
\int_{E} \pi(a) f d m=T_{0}\left(\int_{E} \pi(a) f d k\right)=T_{0}\left(\pi(a) \int_{E} f d k\right)
$$

$$
T_{0}\left(\pi(a) \int_{E} f d k\right)=a T_{0} \int_{E} f d k=a \int_{E} f d m .
$$

Thus $m$ is a modular $\mathscr{A}_{0}$-valued measure with respect to $\pi$.

Let $\mathscr{B}_{n}$ be the collection of all Borel sets $B$ of $E$ such that $k B \in \mathscr{A}_{n}$. Then $L^{\infty}\left(E, \mathscr{B}_{n}, m\right) \cong \mathscr{A}_{n}$ for each $n \geqq 1$. Let $\mathscr{B}_{\infty}$ be the smallest $\sigma$-subalgebra of the Borel sets of $E$ which contains $\bigcup_{n=1}^{\infty} \mathscr{B}_{n}$. Thus $L^{\infty}\left(E, \mathscr{B}_{\infty}, m\right)$ is a Stone subalgebra of $L^{\infty}(E, m) \cong \mathscr{A}_{\infty}$ and contains each of the algebras $\mathscr{A}_{n}(n=1,2, \ldots)$. Thus $L^{\infty}\left(E, \mathscr{B}_{\infty}, m\right) \cong \mathscr{A}_{\infty} \cong L^{\infty}(E, m)$, although $\mathscr{B}_{\infty}$ may not contain all the Borel sets of $E$.

Since $\pi\left[\mathscr{A}_{0}\right] \subset \mathscr{A}_{n}$ for $n \geqq 1$ and $m$ is an $\mathscr{A}_{0}$-valued measure, which is modular with respect to $\pi$, there exists a generalized conditional expectation operator $T_{n}$ mapping $\mathscr{A}_{\infty}$ onto $\mathscr{A}_{n}$. Thus $T_{n}$ is a projection of $\mathscr{A}_{\infty}$ onto $\mathscr{A}_{n}$; if $b$ is a positive element of $\mathscr{A}_{\infty}$ and $T_{n} b=0$, then $b=0 ; T_{n}$ is the unique linear operator from $\mathscr{A}_{\infty}$ into $\mathscr{A}_{n}$ such that for each idempotent $e \in \mathscr{A}_{n}$ and each $z \in \mathscr{A}_{\infty}$ we have $T_{0}\left(e T_{n} z\right)=T_{0}(e z)$. Let $1 \leqq r<n$ and let $e$ be an idempotent of $\mathscr{A}_{r}$ and $z \in \mathscr{A}_{\infty} ;$ then $T_{0}\left(e T_{r} T_{n} z\right)=T_{0}\left(e T_{n} z\right)=T_{0}(e z)$, and so $T_{r} T_{n}=T_{r}$.

It remains to show that, if $z \in \mathscr{A}_{\infty}$, then the order limit LIM $T_{n} z$ exists and equals $z$. Let us identify $\mathscr{A}_{\infty}$ with $C(E)$ so that $z$ and each $T_{n} z(n \geqq 1)$ are continuous functions in $C(E)$. We have from Theorem 1 that there exists a Borel set $B$ such that $m B=0$ and $\lim \left(T_{\mathrm{n}} z\right)(t)$ exists and equals $z(t)$ for each $t \in E-B$. The sequence $\left\{T_{n} z\right\}(n=1,2, \ldots)$ is uniformly bounded because each $T_{n}$ is a positive operator and $T_{n} 1=1$. Since $m B=0$ only if $k B=0$, we have, by the analogue of the Dominated Convergence Theorem proved in [5], that

$$
\operatorname{LIM} \int_{E} T_{n} z d k \text { exists and equals } \int_{E} z d k
$$

Thus LIM $T_{n} z$ exists and equals $z$. 
COROLlary. Let $\left\{\mathscr{A}_{n}\right\}(n=1,2, \ldots)$ be an increasing sequence of Stone subalgebras of a Stone algebra $\mathscr{A}_{\infty}$ such that the smallest Stone subalgebra containing $\bigcup_{n=1}^{\infty} \mathscr{A}_{n}$ is the whole of $\mathscr{A}_{\infty}$. Let $\mathscr{A}_{\infty}$ satisfy the countable chain condition. Let $T_{0}$ be a Moy operator on $\mathscr{A}_{\infty}$ whose range is a subset of $\mathscr{A}_{1}$, and is such that if $b$ is a positive element of $\mathscr{A}_{\infty}$ and $T_{0} b=0$ then $b=0$. Then there exists a sequence of Moy operators $\left\{T_{n}\right\}, n=1,2, \ldots$, such that:

(i) $T_{n}$ is a projection of $\mathscr{A}_{\infty}$ onto $\mathscr{A}_{n}$ for $n \geqq 1$.

(ii) If $b$ is a positive element of $\mathscr{A}_{\infty}$ and $T_{n} b=0$ then $b=0$.

(iii) $T_{r}=T_{r} T_{n}$ for $0 \leqq r<n$.

(iv) For each $z \in \mathscr{A}_{\infty}$ the order limit LIM $T_{n} z$ exists and LIM $T_{n} z=z$.

Proof. Since $\mathscr{A}_{\infty}$ satisfies the countable chain condition, we have that

$$
\mathscr{E}\left(T_{0}\right)=\left\{a \in \mathscr{A}_{\infty}: a T b=T a b \text { for all } b \in \mathscr{A}_{\infty}\right\}
$$

is a Stone subalgebra of $\mathscr{A}_{\infty}$. Let $\mathscr{A}_{0}$ be the smallest Stone subalgebra of $\mathscr{A}_{\infty}$ containing the range of $T_{0}$. Thus $\mathscr{A}_{0} \subset \mathscr{A}_{1}$ and $\mathscr{A}_{0} \subset \mathscr{E}\left(T_{0}\right)$. Let $\pi: \mathscr{A}_{0} \rightarrow \mathscr{A}_{\infty}$ be the natural embedding. Then $T_{0} \pi(a) z=a T_{0} z$ for each $a \in \mathscr{A}_{0}$ and each $z \in \mathscr{A}_{\infty}$. The corollary now follows from Theorem 2.

These methods can be adapted to prove analogous convergence theorems, where instead of $\left\{\mathscr{A}_{n}\right\}(n=1,2, \ldots)$ being monotone increasing it is monotone decreasing and

$$
\pi\left[\mathscr{A}_{0}\right] \subset \bigcap_{n=1}^{\infty} \mathscr{A}_{n}=\mathscr{A}_{\infty} .
$$

In Theorem 1 we required the measure $\rho$ to be modular so as to ensure the existence of the generalized conditional expectations $T_{n}$. We observe that we can dispense with the hypothesis that $\rho$ is modular if we know that the conditional expectation $T_{1}$ of $\mathscr{B}_{1}$ with respect to $\rho$ exists. This is because $T_{1}$ may be regarded as an $L^{\infty}\left(X, \mathscr{B}_{1}, \rho\right)$-valued modular measure and so there is a conditional expectation $T_{n}$ of $\mathscr{B}_{n}$ with respect to $T_{1}$ for each $n$. A straightforward computation shows that $T_{n}$ is the conditional expectation of $\mathscr{B}_{n}$ with respect to $\rho$. The proof, in Theorem 1, that $\left\{T_{n} f\right\}(n=1,2, \ldots)$ converges almost everywhere to $f$ depends only on the existence of the conditional expectations $T_{n}$ and not on the modularity of $\rho$.

\section{REFERENCES}

1. E. Sparre Andersen and B. Jessen, Some limit theorems on set functions, Mat.-Fys. Medd. Danske Vid. Selsk. 25 (1948), No. 5.

2. J. L. Doob, Stochastic processes (Wiley, New York, 1953). 687-689.

3. E. E. Floyd, Boolean algebras with pathological order topologies, Pacific J. Math. 5 (1955),

4. M. H. Stone, Boundedness properties in function lattices, Canad. J. Math. 1 (1949), 176-186.

5. J. D. Maitland Wright, Stone algebra valued measures and integrals, Proc. London Math. Soc.; to appear

6. J. D. Maitland Wright, A Radon-Nikodym theorem for Stone algebra valued measures, Trans. Amer. Math. Soc.; to appear.

7. J. D. Maitland Wright, Applications to averaging operators of the theory of Stone algebra valued measure, Quart. J. Math. Oxford Ser. (2) 19 (1968), 321-331.

\section{St. Carherine's College}

OXFORD 